\title{
Age and Sex Specific Reference Intervals for Modifiable Risk Factors of Cardiovascular Diseases for Gujarati Asian Indians
}

\author{
Sibasis Sahoo, ${ }^{1}$ Komal H. Shah, ${ }^{2}$ Ashwati R. Konat, ${ }^{2}$ \\ Kamal H. Sharma, ${ }^{1}$ and Payal Tripathi ${ }^{3}$ \\ ${ }^{1}$ Department of Cardiology, U. N. Mehta Institute of Cardiology and Research Centre (UNMICRC), Asarwa, \\ Ahmedabad 380016, India \\ ${ }^{2}$ Research Department, U. N. Mehta Institute of Cardiology and Research Centre (UNMICRC), Asarwa, \\ Ahmedabad 380016, India \\ ${ }^{3}$ Pathology Department, U. N. Mehta Institute of Cardiology and Research Centre (UNMICRC), Asarwa, \\ Ahmedabad 380016, India \\ Correspondence should be addressed to Sibasis Sahoo; shasikant_sahoo@yahoo.com
}

Received 30 June 2015; Revised 19 November 2015; Accepted 7 December 2015

Academic Editor: Jose Tellez-Zenteno

Copyright (C) 2015 Sibasis Sahoo et al. This is an open access article distributed under the Creative Commons Attribution License, which permits unrestricted use, distribution, and reproduction in any medium, provided the original work is properly cited.

\begin{abstract}
Objective. We aimed to establish age and sex specific percentile reference data for cardiovascular risk factors such as lipids, sugar, blood pressure, and BMI in apparently healthy and disease-free Gujarati population. Methods. In this cross-sectional study, we enrolled 3265 apparently healthy and disease-free individuals of both genders residing in Gujarat state. Fasting samples of blood were used for biochemical estimations of lipids and sugar. The measurement of BMI and blood pressure was also done according to the standard guidelines. Age and gender specific 5th, 25th, 50th, 75th, 90th, and 95th percentiles were obtained. Results. The mean values of lipids, sugar, blood pressure, and BMI were significantly $(p<0.001)$ higher in males as compared to female population. Age-wise distribution trends showed increase in the risk factors from the 2nd decade until the 5th to 6th decade in most of the cases, where loss of premenopausal protection in females was also observed. Specific trends according to gender and age were observed in percentile values of various parameters. Conclusion. The outcome of current study will contribute significantly to proposing clinically important reference values of various lipids, sugar, blood pressure, and BMI that could be used to screen the asymptomatic Gujarati Indian population with a propensity of developing dyslipidemia, diabetes, blood pressure, and obesity.
\end{abstract}

\section{Introduction}

South Asians, especially Indians, are group of communities bearing genetically diverse ethnic characteristics and are passing through epidemic health shift due to urbanization [1]. This leads to a marked increase in the incidence of noncommunicable diseases such as cardiovascular diseases (CVDs), which are subjected to race-ethnic diversity and are longitudinally related to the classical risk profile of the particular community [2]. Mortality and morbidity statistics of the last few decades had reported substantial increase and regional variation in cardiovascular epidemic trends in India [3]. Hence, it is noteworthy that despite similar risk factor profile the prediction and interpretation of mortality and morbidity in Indians are prone to ethnic and geographic variations. Socioeconomic status and presence of modifiable risk factors such as individual life style factors, lipid levels, diabetes, and hypertension are noted as the proximate/primordial determinants of CVDs [4].

The circulating lipid levels provide substantially good evidence of progressive atherosclerosis in asymptomatic individuals $[5,6]$. Reports from various individual studies have documented steep rise in mean values of Total Cholesterol (TC), Low Density Lipoprotein Cholesterol (LDL-C) and non-High Density Lipoprotein Cholesterol (non-HDL-C), and Triglyceride (TG) and fall in HDL-C in Indians [7]. A population based survey had reported a great degree of variation in lipid associated allele frequencies across multiple 
racial and ethnic population [8]. Moreover, the evaluation of ethnicity based factors on lipid levels in various communities was recommended by Third Report of the National Cholesterol Education Program Expert Panel on Detection, Evaluation, and Treatment of High Blood Cholesterol in Adults (NCEP-ATP III) also [9]. Hence, expert panels from various regions of world have established well-defined guidelines for reference values of lipids, and following this in India also various states have proposed the reference ranges for lipids exclusive for particular geographic and ethnic population [10-12]. In the same manner, racial disparities in diabetes, hypertension, and obesity are reported by many.

Gujarat is a rich state located in the western region of India. Numerous studies conducted on native and migrated Gujarati populations have reported that this community possesses different cardiovascular risk factor profile as compared to other ethnic groups $[13,14]$. Apart from the fact that it has the highest number of newly diagnosed diabetic population, it also has considerably high incidence of smokeless tobacco consumption, obesity, and dyslipidemias [15-17]. The earlier attempt of comparing the mean values of lipid profile components of healthy Gujaratis with other Indian communities showed a great degree of variations between these groups [18]. Moreover, the age and sex specific representative percentile reference data for lipids, sugar, hypertension, and Body Mass Index (BMI) of Gujarati population is lacking. Keeping in mind the lacuna of research, current study was designed to establish the percentile values for serum lipids and sugar, blood pressure, and BMI in healthy Gujarati male and female in order to provide reference data of clinical relevance.

\section{Methods}

2.1. Design and Data Collection. This cross-sectional study was conducted in September 2013 at U. N. Mehta Institute of Cardiology and Research Center which was approved and cleared by institutional ethics committee. In the current investigation, 3265 individuals of both genders, who were apparently healthy, free from any illness and major complications or surgery for the last three months with normal BP, ECG, ECHO, and stress test, were selected. The sample was taken from the self-reported healthy population who visited the hospital during awareness camp. The population was from various regions of Gujarat state. The demographic details of the population such as age, sex, and disease history were recorded for all the individuals. The subjects were asked to relax and then the blood pressure was measured for three times and the average was taken into account. All clinical investigations were conducted according to the principles expressed in the Declaration of Helsinki.

2.2. Laboratory Tests. Fasting serum lipids and glucose concentrations were measured by International Federation of Clinical Chemistry (IFCC) approved enzymatic methods using commercially available kit on autoanalyzer (ARCHITECT PLUS ci4100, Germany). Lipids levels were classified according to the National Cholesterol Education Program (NCEP) and Adult Treatment Panel III (ATP III) guidelines.
Blood pressure of the population was measured according to earlier reported guidelines [19]. Individuals having BMI greater than 30 were considered to be obese as per WHO standards [20].

2.3. Quality Control. The accuracy and precision of laboratory parameters were achieved by participating in the external and internal quality control programs of CMC, Vellore, and Bio-Rad. To maintain the reproducibility and reliability of the test, the quality assessment programs were routinely practiced and accordingly the values were standardized for mean \pm 1 standard deviation (SD). For internal quality control, the accuracy of reports was tested by retesting the same sample within the hospital. Performance of all results was done following quality control program based on west grade QC rule like $1-2 \mathrm{~s}$ as warning rule and $2-2 \mathrm{~s}$, 1$3 \mathrm{~s}$, and $1-4 \mathrm{~s}$ as rejection rule. Test performance was started with acceptable QC range. In case of rejection (QC outlier), recalibration and use of fresh control analysis were done. For external quality control, the sample was sent to reputed NABL accredited laboratories for interlab comparison and verification of accuracy of the reports. In addition, the participation in periodic EQAS Programs for Haematology, Clinical Chemistry and Immunoassay was done on the basis of AIIMS, Delhi, Bio-Rad, USA, respectively. The data obtained from everyday quality control check was expressed in terms of SD and Coefficient of Variation (CV) and is presented in Table 1.

2.4. Statistical Analysis. All collected data were analyzed by SPSS v. 20 (Chicago, IL, USA). Distribution analysis showed that most of the parameters follow non-Gaussian distribution. Mean, median, and percentile data of 5th, 25th, 50th, 75th, and 95th were calculated for the values of lipids, glucose, blood pressure, and BMI.

\section{Results}

The overall results obtained from the current investigation are presented in Table 2 as mean \pm SD and median of lipid profile, glucose, blood pressure, and BMI in Gujarati Indian population.

Our results showed mean \pm SD of cholesterol to be $185.33 \pm 39.24 \mathrm{mg} / \mathrm{dL}$ and Triglyceride in the studied population was found to be $115.59 \pm 63.47 \mathrm{mg} / \mathrm{dL}$. The mean $\pm \mathrm{SD}$ was $118.5 \pm 33.45 \mathrm{mg} / \mathrm{dL}$ for LDL-C and $43.7 \pm$ $9.87 \mathrm{mg} / \mathrm{dL}$ for HDL-C and $23.11 \pm 12.69 \mathrm{mg} / \mathrm{dL}$ for VLDL. The mean reference values obtained for TL, LDL-C/HDL$\mathrm{C}$, and TC/HDL-C were $644.62 \pm 88.02,2.84 \pm 1.02$, and $4.42 \pm 1.4 \mathrm{mg} / \mathrm{dL}$, respectively. In the current study cohort females showed superior lipid profile (TC: $181.55 \pm 38.93$ versus $188.48 \pm 39.2$; TG: $100.63 \pm 48.18$ versus $127.91 \pm 71.44$; HDL-C: $47.52 \pm 9.68$ versus $40.56 \pm 8.81$; LDL-C: $113.89 \pm 33.54$ versus $122.34 \pm 32.89$; VLDL: $20.12 \pm 9.63$ versus $25.58 \pm 14.28$; TL: $629.72 \pm 75.45$ versus $656.96 \pm 95.46$; LDL-C/HDL-C: $2.49 \pm 0.88$ versus $3.12 \pm 1.01$; TC/HDL-C: $3.94 \pm 1.07$ versus $4.81 \pm 1.46)$, glucose $(83.08 \pm 21.49$ versus $88.64 \pm 25.12)$, blood pressure (SBP: $126.47 \pm 18.87$ versus $133.74 \pm 17.97$; 
TABLE 1: Comparison of quality control material at levels I and II from Bio-Rad with the mean and percentage of Coefficient of Variations obtained from laboratory.

\begin{tabular}{|c|c|c|c|c|c|c|}
\hline \multirow{2}{*}{ Analyte } & \multicolumn{2}{|c|}{ Reference mean } & \multicolumn{2}{|c|}{ Mean obtained } & \multicolumn{2}{|c|}{$\%$ of $\mathrm{CV}$} \\
\hline & Level I & Level II & Level I & Level II & Level I & Level II \\
\hline $\mathrm{TC}(\mathrm{mg} / \mathrm{dL})$ & 249.6 & 104.4 & 250.1 & 103.9 & 3.4 & 0.92 \\
\hline $\mathrm{TG}(\mathrm{mg} / \mathrm{dL})$ & 182.5 & 95.9 & 182.3 & 95.2 & 1.4 & 1.06 \\
\hline HDL-C (mg/dL) & 65.4 & 26.9 & 65.2 & 26.5 & 2.5 & 1.96 \\
\hline Blood sugar (mg/dL) & 297.9 & 81.8 & 297.6 & 81.5 & 3.3 & 3.3 \\
\hline
\end{tabular}

Level I: Bio-Rad control (abnormal); level II: Bio-Rad control (normal); CV: Coefficient of Variation; TC: Total Cholesterol; TG: Triglyceride; HDL-C: High Density Lipoprotein Cholesterol.

TABLE 2: Mean and median values of lipid profile, sugar, blood pressure, and BMI in Gujarati population.

\begin{tabular}{|c|c|c|c|}
\hline Variable & Total & Male & Female \\
\hline \multicolumn{4}{|l|}{$\mathrm{TC}(\mathrm{mg} / \mathrm{dL})$} \\
\hline Mean \pm SD & $185.33 \pm 39.24$ & $188.48 \pm 39.20$ & $181.55 \pm 38.93$ \\
\hline Median & 182 & 187 & 178 \\
\hline \multicolumn{4}{|l|}{ TG (mg/dL) } \\
\hline Mean \pm SD & $115.59 \pm 63.47$ & $127.91 \pm 71.44$ & $100.63 \pm 48.18$ \\
\hline Median & 99 & 110 & 88 \\
\hline \multicolumn{4}{|c|}{ HDL-C (mg/dL) } \\
\hline Mean \pm SD & $43.7 \pm 9.87$ & $40.56 \pm 8.81$ & $47.52 \pm 9.68$ \\
\hline Median & 43 & 40 & 47 \\
\hline \multicolumn{4}{|l|}{ LDL-C (mg/dL) } \\
\hline Mean \pm SD & $118.5 \pm 33.45$ & $122.34 \pm 32.89$ & $113.89 \pm 33.54$ \\
\hline Median & 116 & 121.2 & 110 \\
\hline \multicolumn{4}{|l|}{ LDL-C/HDL-C } \\
\hline Mean \pm SD & $2.84 \pm 1.02$ & $3.12 \pm 1.01$ & $2.49 \pm 0.88$ \\
\hline Median & 2.71 & 3.05 & 2.37 \\
\hline \multicolumn{4}{|l|}{ TC/HDL-C } \\
\hline Mean \pm SD & $4.42 \pm 1.40$ & $4.81 \pm 1.46$ & $3.94 \pm 1.07$ \\
\hline Median & 4.26 & 4.68 & 3.77 \\
\hline \multicolumn{4}{|l|}{ VLDL (mg/dL) } \\
\hline Mean \pm SD & $23.11 \pm 12.69$ & $25.58 \pm 14.28$ & $20.12 \pm 9.63$ \\
\hline Median & 19.8 & 22 & 17.6 \\
\hline \multicolumn{4}{|l|}{$\mathrm{TL}(\mathrm{mg} / \mathrm{dL})$} \\
\hline Mean \pm SD & $644.62 \pm 88.02$ & $656.96 \pm 95.46$ & $629.72 \pm 75.45$ \\
\hline Median & 629 & 641.5 & 617 \\
\hline \multicolumn{4}{|c|}{ Blood sugar (mg/dL) } \\
\hline Mean \pm SD & $86.13 \pm 23.71$ & $88.64 \pm 25.12$ & $83.08 \pm 21.49$ \\
\hline Median & 83 & 85 & 81 \\
\hline \multicolumn{4}{|l|}{$\mathrm{SBP}(\mathrm{mm} / \mathrm{Hg})$} \\
\hline Mean \pm SD & $130.45 \pm 18.73$ & $133.74 \pm 17.97$ & $126.47 \pm 18.87$ \\
\hline Median & 130 & 130 & 122 \\
\hline \multicolumn{4}{|l|}{$\mathrm{DBP}(\mathrm{mm} / \mathrm{Hg})$} \\
\hline Mean \pm SD & $82.57 \pm 10.61$ & $84.14 \pm 10.55$ & $80.67 \pm 10.37$ \\
\hline Median & 80 & 82 & 80 \\
\hline \multicolumn{4}{|l|}{ BMI $\left(\mathrm{kg} / \mathrm{m}^{2}\right)$} \\
\hline Mean \pm SD & $26.87 \pm 66.52$ & $27.09 \pm 44.67$ & $26.61 \pm 85.88$ \\
\hline Median & 24.12 & 24.16 & 24.05 \\
\hline
\end{tabular}

TC: Total Cholesterol; TG: Triglycerides; LDL-C: Low Density Lipoprotein Cholesterol; HDL-C: High Density Lipoprotein Cholesterol; VLDL: Very Low Density Lipoprotein; TL: Total Lipid; SBP: Systolic Blood Pressure; DBP: Diastolic Blood Pressure; BMI: Body Mass Index.

Data was expressed as mean \pm SD. Significance was obtained between males and females using Mann-Whitney $U$ Test. Level of significance was accepted at $p<0.05$ level. 
TABLE 3: Percentile values of lipid profile, sugar, blood pressure, and BMI in population.

\begin{tabular}{|c|c|c|c|c|c|c|c|c|}
\hline & & $n$ & 5th percentile & 25th percentile & 50th percentile & 75th percentile & 95th percentile & 97th percentile \\
\hline \multirow{2}{*}{$\mathrm{TC}(\mathrm{mg} / \mathrm{dL})$} & Men & 1789 & 128 & 161 & 187 & 213.5 & 255 & 268.3 \\
\hline & Women & 1476 & 127 & 154.25 & 178 & 204 & 250 & 262 \\
\hline \multirow{2}{*}{ TG (mg/dL) } & Men & 1789 & 59 & 83 & 110 & 152 & 257.5 & 293.5 \\
\hline & Women & 1476 & 50.85 & 68 & 88 & 119 & 194.3 & 214 \\
\hline \multirow{2}{*}{ HDL-C (mg/dL) } & Men & 1789 & 29 & 35 & 40 & 45 & 55 & 58.3 \\
\hline & Women & 1476 & 33 & 41 & 47 & 53 & 65 & 67 \\
\hline \multirow{2}{*}{ LDL-C (mg/dL) } & Men & 1789 & 71 & 99 & 121 & 142 & 178 & 188.3 \\
\hline & Women & 1476 & 66.85 & 89 & 110 & 134 & 174 & 183 \\
\hline \multirow{2}{*}{ LDL-C/HDL-C } & Men & 1789 & 1.64 & 2.4 & 3.05 & 3.76 & 4.8 & 5.04 \\
\hline & Women & 1476 & 1.25 & 1.83 & 2.37 & 3.02 & 4.08 & 4.39 \\
\hline \multirow{2}{*}{ TC/HDL-C } & Men & 1789 & 2.97 & 3.87 & 4.68 & 5.58 & 7.05 & 7.47 \\
\hline & Women & 1476 & 2.5 & 3.16 & 3.77 & 4.6 & 5.91 & 6.26 \\
\hline \multirow{2}{*}{ VLDL (mg/dL) } & Men & 1789 & 12 & 17 & 22 & 30 & 51.5 & 58.3 \\
\hline & Women & 1476 & 10 & 14 & 18 & 24 & 39 & 43 \\
\hline \multirow{2}{*}{$\mathrm{TL}(\mathrm{mg} / \mathrm{dL})$} & Men & 1789 & 540 & 592 & 641 & 701 & 830 & 865 \\
\hline & Women & 1476 & 531 & 577 & 617 & 672 & 772.15 & 799.69 \\
\hline \multirow{2}{*}{ Blood sugar $(\mathrm{mg} / \mathrm{dL})$} & Men & 1789 & 65 & 76 & 85 & 94 & 120 & 139 \\
\hline & Women & 1476 & 62 & 73 & 81 & 89 & 108 & 115 \\
\hline \multirow{2}{*}{$\mathrm{SBP}(\mathrm{mm} / \mathrm{Hg})$} & Men & 1789 & 110 & 120 & 130 & 142 & 168 & 176 \\
\hline & Women & 1476 & 100 & 112 & 122 & 136 & 162 & 176 \\
\hline \multirow{2}{*}{$\mathrm{DBP}(\mathrm{mm} / \mathrm{Hg})$} & Men & 1789 & 70 & 80 & 82 & 90 & 100 & 100.6 \\
\hline & Women & 1476 & 70 & 70 & 80 & 88 & 100 & 100 \\
\hline \multirow{2}{*}{ BMI $\left(\mathrm{kg} / \mathrm{m}^{2}\right)$} & Men & 1789 & 17 & 22 & 24 & 27 & 32 & 34 \\
\hline & Women & 1476 & 17 & 20 & 24 & 27 & 33 & 35 \\
\hline
\end{tabular}

TC: Total Cholesterol; TG: Triglycerides; HDL-C: High Density Lipoprotein Cholesterol; LDL-C: Low Density Lipoprotein Cholesterol; VLDL: Very Low Density Lipoprotein; TL: Total Lipid; SBP: Systolic Blood Pressure; DBP: Diastolic Blood Pressure; BMI: Body Mass Index. Data are expressed as the 5th to 97th percentile of median.

DBP: $80.67 \pm 10.37$ versus $84.14 \pm 10.55)$, and BMI $(26.61 \pm$ 85.88 versus $27.09 \pm 44.67)$ as compared to males, which was highly significant $(p<0.001$; Table 2$)$.

The percentile limits from 5 th to 95 th for various lipids, glucose, and blood pressure according to gender tabulated in Table 3 show higher cutoff values of all the variables in males as compared to females except for slight elevation in BMI levels in higher percentile values. Age-wise distribution trends analysis showed steep increase in the levels of lipids, glucose, blood pressure, and obesity from the 2 nd decade till the 5 th to 6 th decade in most of the cases (Table 4), indicating natural ageing itself as one of the risk factors. In contrast to others, the level of HDL-C was found to increase with the advancing of age. The 5th to the 95th percentile values of lipids, glucose, SBP, DBP, and BMI in various age groups were also analyzed (Table 5). Most of the variables achieved stable levels after the 5 th decade and in some cases a decline was observed after the 6 th decade of life.

Comparative examination of reference values established for various communities based on the population mean has been presented in Table 6. We have observed that the TC of Gujarati population had the third highest $(185.33 \pm$ $39.245 \mathrm{mg} / \mathrm{dL}$ ) mean value in comparison to other Indian populations, whereas LDL-C had the third highest (118.5 \pm 33.46) mean value amongst all including Venezuelan and
Nepali populations. The level of good cholesterol, HDL-C, was lowest $(43.70 \pm 9.870)$ in Gujarati population.

\section{Discussion}

The need for establishment of different reference intervals of lipids, sugar, blood pressure, and obesity in various community could be justified by the fact that these risk factors are subjected to racial and geographic variations with life style changes, demographic shifts, epidemiological transition, and urbanization playing a prime role. Moreover, Indians possess some of the unique characteristics such as higher predisposition to the development of type 2 diabetes mellitus, excess visceral fat and centripetal obesity, and smaller body size, which needs to be considered before screening the patients with any guidelines formerly standardized on western population [21]. Along with the "genetically deformed life style," other modifiable risk factors of CVD such as dyslipidemia, diabetes, hypertension, and obesity are increasing in Gujarati population $[22,23]$. A physically inactive life style and diet rich in oil and sugar have made Gujarat the second largest contributor of Indian diabetic population. This ethnic group is highly prone to develop and suffer from premature vascular ageing as their heart age is 6.7 years higher than their chronological age with dyslipidemia, 
TABLE 4: Decade-wise percentile values of lipid profile, sugar, blood pressure, and BMI in Gujarati population.

\begin{tabular}{|c|c|c|c|c|c|c|c|}
\hline & & $20-29$ & $30-39$ & $40-49$ & $50-59$ & $60-69$ & $>70$ \\
\hline \multirow{3}{*}{$\mathrm{TC}(\mathrm{mg} / \mathrm{dL})$} & Total & $161.29 \pm 30.64$ & $181.18 \pm 178$ & $193.10 \pm 37.01$ & $201.40 \pm 38.81$ & $201.31 \pm 41.98$ & $197.98 \pm 37.94$ \\
\hline & Male & $165.93 \pm 32.64$ & $187.38 \pm 37.99$ & $193.43 \pm 38.89$ & $197.94 \pm 38.69$ & $195.83 \pm 40.28$ & $193.91 \pm 33.42$ \\
\hline & Female & $158.15 \pm 28.83$ & $172.89 \pm 30.15$ & $192.70 \pm 34.64$ & $206.90 \pm 38.51$ & $213.04 \pm 43.49$ & $219.22 \pm 53.58$ \\
\hline \multirow{3}{*}{ TG (mg/dL) } & Total & $89.7 \pm 42.60$ & $116.05 \pm 71.59$ & $122.85 \pm 66.11$ & $131.56 \pm 65.81$ & $127.38 \pm 58.36$ & $128.28 \pm 60.26$ \\
\hline & Male & $106.09 \pm 50.87$ & $131.09 \pm 82.10$ & $133.23 \pm 76.71$ & $138.90 \pm 73.11$ & $124.34 \pm 40.28$ & $124.65 \pm 61.63$ \\
\hline & Female & $78.63 \pm 31.50$ & $95.95 \pm 47.68$ & $110.30 \pm 47.54$ & $119.87 \pm 50.27$ & $134.21 \pm 62.95$ & $147.22 \pm 51.47$ \\
\hline \multirow{3}{*}{ HDL-C (mg/dL) } & Total & $45.43 \pm 10.56$ & $42.18 \pm 8.72$ & $43.37 \pm 9.34$ & $43.44 \pm 9.24$ & $43.93 \pm 11.93$ & $44.12 \pm 10.39$ \\
\hline & Male & $40.30 \pm 8.22$ & $39.56 \pm 7.62$ & $40.15 \pm 7.89$ & $40.87 \pm 8.49$ & $42.58 \pm 12.88$ & $43.08 \pm 9.91$ \\
\hline & Female & $48.98 \pm 10.57$ & $45.69 \pm 8.89$ & $47.26 \pm 9.48$ & $47.51 \pm 8.95$ & $46.97 \pm 8.75$ & $49.55 \pm 11.72$ \\
\hline \multirow{3}{*}{ LDL-C (mg/dL) } & Total & $97.91 \pm 26.15$ & $115.78 \pm 29.61$ & $125.16 \pm 31.80$ & $131.64 \pm 33.89$ & $131.72 \pm 35.81$ & $128.2 \pm 30.16$ \\
\hline & Male & $104.41 \pm 27.69$ & $121.59 \pm 31.38$ & $126.63 \pm 32.55$ & $129.28 \pm 33.62$ & $128.38 \pm 33.81$ & $125.89 \pm 26.86$ \\
\hline & Female & $93.52 \pm 24.12$ & $108.01 \pm 25.09$ & $123.37 \pm 30.83$ & $135.41 \pm 34.13$ & $139.22 \pm 39.11$ & $140.22 \pm 43.72$ \\
\hline \multirow{3}{*}{ LDL-C/HDL-C } & Total & $2.28 \pm 0.87$ & $2.85 \pm 2.71$ & $3.00 \pm 0.94$ & $3.15 \pm 1.06$ & $3.13 \pm 3.04$ & $3.0 \pm 0.82$ \\
\hline & Male & $2.70 \pm 0.90$ & $3.16 \pm 0.95$ & $3.24 \pm 0.95$ & $3.28 \pm 1.13$ & $3.17 \pm 1.03$ & $3.04 \pm 0.87$ \\
\hline & Female & $2.00 \pm 0.74$ & $2.44 \pm 0.70$ & $2.70 \pm 0.84$ & $2.94 \pm 0.91$ & $3.04 \pm 0.94$ & $2.82 \pm 0.53$ \\
\hline \multirow{3}{*}{ TC/HDL-C } & Total & $3.72 \pm 1.08$ & $4.45 \pm 1.19$ & $4.62 \pm 1.20$ & $4.84 \pm 1.77$ & $4.76 \pm 1.24$ & $4.64 \pm 1.11$ \\
\hline & Male & $4.26 \pm 1.12$ & $4.87 \pm 1.22$ & $4.95 \pm 1.22$ & $5.06 \pm 2.06$ & $4.81 \pm 1.29$ & $4.68 \pm 1.19$ \\
\hline & Female & $3.35 \pm 0.88$ & $3.89 \pm 0.88$ & $4.21 \pm 1.02$ & $4.48 \pm 1.08$ & $4.65 \pm 1.12$ & $4.44 \pm 0.60$ \\
\hline \multirow{3}{*}{ VLDL (mg/dL) } & Total & $17.94 \pm 8.52$ & $23.21 \pm 14.31$ & $24.571 \pm 13.22$ & $26.31 \pm 13.16$ & $25.74 \pm 11.67$ & $25.65 \pm 12.05$ \\
\hline & Male & $21.21 \pm 10.17$ & $26.21 \pm 16.42$ & $26.64 \pm 15.34$ & $27.78 \pm 14.62$ & $24.86 \pm 11.21$ & $24.93 \pm 12.32$ \\
\hline & Female & $15.72 \pm 6.30$ & $19.19 \pm 9.53$ & $22.06 \pm 9.50$ & $23.97 \pm 10.05$ & $26.84 \pm 12.59$ & $29.44 \pm 10.29$ \\
\hline \multirow{3}{*}{ TL (mg/dL) } & Total & $596.42 \pm 63.56$ & $639.4 \pm 93.55$ & $659.33 \pm 86.84$ & $676.40 \pm 85.69$ & $672.44 \pm 85.32$ & $670.39 \pm 82.16$ \\
\hline & Male & $612.33 \pm 72.96$ & $658.04 \pm 105.79$ & $666.82 \pm 99.99$ & $677.72 \pm 92.57$ & $662.75 \pm 82.91$ & $661.65 \pm 79.75$ \\
\hline & Female & $585.68 \pm 53.84$ & $614.5 \pm 66.55$ & $650.26 \pm 66.62$ & $674.30 \pm 73.77$ & $694.23 \pm 87.09$ & $716.00 \pm 83.97$ \\
\hline \multirow{3}{*}{ Blood sugar (mg/dL) } & Total & $76.93 \pm 11.06$ & $82.80 \pm 15.86$ & $88.816 \pm 23.10$ & $93.49 \pm 30.84$ & $94.55 \pm 36.14$ & $89.39 \pm 16.43$ \\
\hline & Male & $79.23 \pm 11.02$ & $85.16 \pm 17.77$ & $87.00 \pm 17.68$ & $95.61 \pm 33.78$ & $92.48 \pm 26.06$ & $90.85 \pm 17.15$ \\
\hline & Female & $75.37 \pm 10.81$ & $79.64 \pm 12.20$ & $87.00 \pm 17.68$ & $90.12 \pm 25.27$ & $99.21 \pm 52.04$ & $81.77 \pm 9.29$ \\
\hline \multirow{3}{*}{ SBP (mm/Hg) } & Total & $120.5 \pm 14.72$ & $127.14 \pm 16.49$ & $133.28 \pm 18.13$ & $137.85 \pm 19.06$ & $139.53 \pm 36.14$ & $138.25 \pm 16.36$ \\
\hline & Male & $128.23 \pm 15.42$ & $129.95 \pm 15.85$ & $134.44 \pm 18.36$ & $137.59 \pm 18.35$ & $139.76 \pm 20.43$ & $137.31 \pm 15.28$ \\
\hline & Female & $115.28 \pm 11.62$ & $123.37 \pm 16.60$ & $131.87 \pm 17.77$ & $138.27 \pm 20.20$ & $139.01 \pm 20.91$ & $143.11 \pm 21.61$ \\
\hline \multirow{3}{*}{$\mathrm{DBP}(\mathrm{mm} / \mathrm{Hg})$} & Total & $77.40 \pm 9.04$ & $81.70 \pm 9.90$ & $84.30 \pm 9.92$ & $86.25 \pm 11.07$ & $85.62 \pm 11.66$ & $83.28 \pm 8.75$ \\
\hline & Male & $80.77 \pm 8.46$ & $83.02 \pm 10.02$ & $84.90 \pm 9.83$ & $86.31 \pm 11.88$ & $85.98 \pm 12.13$ & $83.06 \pm 8.95$ \\
\hline & Female & $75.12 \pm 8.71$ & $79.94 \pm 9.47$ & $83.58 \pm 9.99$ & $86.15 \pm 9.68$ & $84.80 \pm 10.54$ & $84.44 \pm 7.98$ \\
\hline \multirow{3}{*}{ BMI $\left(\mathrm{kg} / \mathrm{m}^{2}\right)$} & Total & $24.78 \pm 42.60$ & $24.40 \pm 5.00$ & $27.52 \pm 44.00$ & $30.98 \pm 131.38$ & $25.42 \pm 6.32$ & $36.99 \pm 94.84$ \\
\hline & Male & $29.48 \pm 66.63$ & $24.33 \pm 4.64$ & $28.94 \pm 59.24$ & $25.23 \pm 4.46$ & $24.81 \pm 4.20$ & $39.40 \pm 103.51$ \\
\hline & Female & $21.60 \pm 4.55$ & $24.50 \pm 5.46$ & $25.82 \pm 5.70$ & $40.12 \pm 211.5$ & $26.79 \pm 9.39$ & $24.39 \pm 4.70$ \\
\hline
\end{tabular}

TC: Total Cholesterol; TG: Triglycerides; HDL-C: High Density Lipoprotein Cholesterol; LDL-C: Low Density Lipoprotein Cholesterol; VLDL: Very Low Density Lipoprotein; TL: Total Lipid; SBP: Systolic Blood Pressure; DBP: Diastolic Blood Pressure; BMI: Body Mass Index.

Data are expressed as mean \pm SD.

hypertension, increased waist circumference, and diabetes as the involved factors [17]. Hence, it is of utmost importance to standardize the reference range of CVD contributing risk factor in Gujaratis. The first attempt to establish normal percentile values of serum lipid levels in healthy, vegetarian Gujarati population was done by Jhala et al. in 1998 [18]. The study showed natural ageing and gender specific disturbance in lipid profile. The author had reappraised the lipid values of the same community in 2009 [24] and compared the data with other populations from different states of India showing variation in lipid profile of Gujaratis as compared to others.
In spite of the significant contribution of both studies in lipid reference range establishment, the findings lacked percentile values specific to various age groups and gender. Moreover, none of the studies has established reference range for blood glucose, blood pressure, and BMI in healthy, adult Gujarati population.

To the best of our knowledge, this is the first ever large study providing representative age and gender specific percentile values for serum lipids, glucose, blood pressure, and BMI in healthy Gujarati Indians. Contrary to earlier reports where no substantial sex specific difference was noted 
TABLE 5: Decade-wise percentile values of lipid profile, sugar, blood pressure, and BMI in Gujarati population.

\begin{tabular}{|c|c|c|c|c|c|c|c|c|}
\hline & Age in years & $n$ & 5th percentile & 25th percentile & 50th percentile & 75th percentile & 95th percentile & 97th percentile \\
\hline \multirow{6}{*}{$\mathrm{TC}(\mathrm{mg} / \mathrm{dL})$} & $20-29$ & 796 & 118 & 140.25 & 157 & 179.75 & 216 & 228.09 \\
\hline & $30-39$ & 653 & 128 & 157.5 & 178 & 201 & 242.3 & 254.38 \\
\hline & $40-49$ & 844 & 138 & 169 & 190 & 213 & 256.5 & 276.3 \\
\hline & $50-59$ & 627 & 140 & 175 & 200 & 226 & 270 & 278 \\
\hline & $60-69$ & 289 & 132.5 & 174 & 198 & 228.5 & 274 & 288.5 \\
\hline & $>70$ & 56 & 127.85 & 173.5 & 202.5 & 220.25 & 254.3 & 275.43 \\
\hline \multirow{6}{*}{ TG (mg/dL) } & $20-29$ & 796 & 47.85 & 63 & 77 & 102.75 & 178.15 & 202.09 \\
\hline & $30-39$ & 653 & 52 & 72 & 98 & 141 & 230.3 & 278 \\
\hline & $40-49$ & 844 & 62 & 83 & 106 & 142.75 & 231.75 & 264.3 \\
\hline & $50-59$ & 627 & 63.4 & 88 & 115 & 153 & 271 & 292.8 \\
\hline & $60-69$ & 289 & 62.5 & 89 & 112 & 154 & 241.5 & 274.5 \\
\hline & $>70$ & 56 & 59 & 74.75 & 116.5 & 172.75 & 240.1 & 284.66 \\
\hline \multirow{6}{*}{ HDL-C (mg/dL) } & $20-29$ & 796 & 30 & 38 & 44 & 52 & 65 & 67.09 \\
\hline & $30-39$ & 653 & 29 & 36 & 42 & 47 & 58 & 60 \\
\hline & $40-49$ & 844 & 31 & 37 & 42 & 49 & 61 & 63 \\
\hline & $50-59$ & 627 & 30 & 38 & 42 & 49 & 61 & 63 \\
\hline & $60-69$ & 289 & 30 & 37 & 42 & 49.5 & 60 & 63.3 \\
\hline & $>70$ & 56 & 30.85 & 36.25 & 41.5 & 51.25 & 64.15 & 66.74 \\
\hline \multirow{6}{*}{ LDL-C (mg/dL) } & $20-29$ & 796 & 60 & 81 & 93 & 113 & 146 & 158 \\
\hline & $30-39$ & 653 & 71 & 94 & 114 & 133 & 169 & 178.38 \\
\hline & $40-49$ & 844 & 77.25 & 104 & 123 & 143 & 179 & 185 \\
\hline & $50-59$ & 627 & 77 & 109 & 131 & 153 & 185.6 & 200 \\
\hline & $60-69$ & 289 & 72.5 & 107.5 & 131 & 155 & 195 & 203 \\
\hline & $>70$ & 56 & 80.25 & 111 & 127 & 146.75 & 177.35 & 196.6 \\
\hline \multirow{6}{*}{ LDL-C/HDL-C } & $20-29$ & 796 & 1.184 & 1.645 & 2.109 & 2.747 & 3.979 & 4.311 \\
\hline & $30-39$ & 653 & 1.573 & 2.185 & 2.717 & 3.414 & 4.498 & 4.792 \\
\hline & $40-49$ & 844 & 1.626 & 2.327 & 2.89 & 3.603 & 4.63 & 4.976 \\
\hline & $50-59$ & 627 & 1.719 & 2.476 & 3.045 & 3.711 & 4.871 & 5.249 \\
\hline & $60-69$ & 289 & 1.707 & 2.316 & 3.047 & 3.882 & 4.956 & 5.037 \\
\hline & $>70$ & 56 & 1.692 & 2.399 & 2.975 & 3.429 & 4.686 & 4.913 \\
\hline \multirow{6}{*}{ TC/HDL-C } & $20-29$ & 796 & 2.359 & 2.924 & 3.474 & 4.31 & 5.74 & 6.195 \\
\hline & $30-39$ & 653 & 2.842 & 3.562 & 4.315 & 5.164 & 6.78 & 7.395 \\
\hline & $40-49$ & 844 & 2.935 & 3.729 & 4.477 & 5.353 & 6.61 & 7.276 \\
\hline & $50-59$ & 627 & 3.116 & 3.914 & 4.666 & 5.468 & 7 & 7.302 \\
\hline & $60-69$ & 289 & 3.008 & 3.805 & 4.738 & 5.602 & 6.88 & 7.278 \\
\hline & $>70$ & 56 & 2.974 & 3.868 & 4.361 & 5.373 & 6.91 & 7.52 \\
\hline \multirow{6}{*}{ VLDL (mg/dL) } & $20-29$ & 796 & 9.85 & 13 & 15 & 20.75 & 36 & 40.09 \\
\hline & $30-39$ & 653 & 10 & 14.5 & 20 & 28 & 46 & 55.76 \\
\hline & $40-49$ & 844 & 12 & 17 & 21 & 28.75 & 46 & 53 \\
\hline & $50-59$ & 627 & 13 & 18 & 23 & 31 & 54.6 & 58.16 \\
\hline & $60-69$ & 289 & 12.5 & 18 & 22 & 31 & 48 & 55.3 \\
\hline & $>70$ & 56 & 12 & 15 & 23.5 & 34.75 & 48.05 & 57.19 \\
\hline \multirow{6}{*}{$\mathrm{TL}(\mathrm{mg} / \mathrm{dL})$} & $20-29$ & 796 & 520 & 555 & 585 & 623 & 722 & 763.09 \\
\hline & $30-39$ & 653 & 533 & 579 & 623 & 677 & 791.9 & 849.9 \\
\hline & $40-49$ & 844 & 560 & 605.25 & 646 & 691 & 799.75 & 848.3 \\
\hline & $50-59$ & 627 & 563.4 & 618 & 665 & 714 & 846.6 & 868.8 \\
\hline & $60-69$ & 289 & 555.5 & 614 & 662 & 714 & 839 & 895 \\
\hline & $>70$ & 56 & 544.55 & 598.75 & 677.5 & 729.75 & 831.65 & 859.45 \\
\hline
\end{tabular}


TABLE 5: Continued.

\begin{tabular}{|c|c|c|c|c|c|c|c|c|}
\hline & Age in years & $n$ & 5th percentile & 25th percentile & 50th percentile & 75th percentile & 95th percentile & 97th percentile \\
\hline \multirow{6}{*}{ Blood sugar $(\mathrm{mg} / \mathrm{dL})$} & $20-29$ & 796 & 60 & 70 & 77 & 84 & 95 & 98.09 \\
\hline & $30-39$ & 652 & 64 & 74 & 81 & 89 & 102 & 112 \\
\hline & $40-49$ & 844 & 67 & 78 & 85 & 94 & 116 & 137.95 \\
\hline & $50-59$ & 627 & 66 & 79 & 88 & 98 & 134.2 & 178.96 \\
\hline & $60-69$ & 289 & 65 & 78 & 89 & 99.5 & 146 & 155.9 \\
\hline & $>70$ & 56 & 69.7 & 79 & 86.5 & 94 & 130.65 & 143.77 \\
\hline \multirow{6}{*}{ SBP (mm/Hg) } & $20-29$ & 796 & 100 & 110 & 120 & 130 & 148 & 152 \\
\hline & $30-39$ & 653 & 104 & 116 & 126 & 136 & 154 & 164 \\
\hline & $40-49$ & 844 & 110 & 120 & 130 & 142 & 169.75 & 176 \\
\hline & $50-59$ & 627 & 110 & 126 & 138 & 150 & 170 & 180 \\
\hline & $60-69$ & 289 & 110 & 126 & 140 & 150 & 179 & 182.6 \\
\hline & $>70$ & 56 & 109.1 & 128 & 140 & 150 & 164.7 & 180 \\
\hline \multirow{6}{*}{$\mathrm{DBP}(\mathrm{mm} / \mathrm{Hg})$} & $20-29$ & 796 & 68 & 70 & 80 & 80 & 90 & 96 \\
\hline & $30-39$ & 653 & 70 & 76 & 80 & 90 & 100 & 100 \\
\hline & $40-49$ & 844 & 70 & 80 & 84 & 90 & 100 & 102 \\
\hline & $50-59$ & 627 & 70 & 80 & 86 & 90 & 100 & 100.32 \\
\hline & $60-69$ & 289 & 70 & 80 & 84 & 90 & 100 & 105.8 \\
\hline & $>70$ & 56 & 67.4 & 80 & 82 & 90 & 100 & 100 \\
\hline \multirow{6}{*}{ BMI $\left(\mathrm{kg} / \mathrm{m}^{2}\right)$} & $20-29$ & 796 & 15.93 & 18.42 & 21.35 & 24.82 & 30.43 & 31.96 \\
\hline & $30-39$ & 653 & 17.45 & 21.35 & 23.93 & 26.65 & 32.11 & 33.92 \\
\hline & $40-49$ & 844 & 17.88 & 22.37 & 25 & 28.14 & 33.69 & 35.3 \\
\hline & $50-59$ & 627 & 19.22 & 22.78 & 25.51 & 28.22 & 33.33 & 34.66 \\
\hline & $60-69$ & 289 & 18.45 & 22.28 & 25.06 & 27.51 & 32.34 & 34.25 \\
\hline & $>70$ & 56 & 17.86 & 21.11 & 23.44 & 26.75 & 33.67 & 35.54 \\
\hline
\end{tabular}

TC: Total Cholesterol; TG: Triglycerides; HDL-C: High Density Lipoprotein Cholesterol; LDL-C: Low Density Lipoprotein Cholesterol; VLDL: Very Low Density Lipoprotein; TL: Total Lipid; SBP: Systolic Blood Pressure; DBP: Diastolic Blood Pressure; BMI: Body Mass Index. Data are expressed as the 5th to 97th percentile of median.

TABLE 6: Comparison between mean lipid values of different studies.

\begin{tabular}{lcccccccc}
\hline & $N$ & TC $(\mathrm{mg} / \mathrm{dL})$ & TG $(\mathrm{mg} / \mathrm{dL})$ & HDL-C $(\mathrm{mg} / \mathrm{dL})$ & LDL-C $(\mathrm{mg} / \mathrm{dL})$ & LDL/HDL & TC/HDL & VLDL $(\mathrm{mg} / \mathrm{dL})$ \\
\hline Our study & 3265 & $185.33 \pm 39.245$ & $115.6 \pm 33.460$ & $43.70 \pm 9.870$ & $118.50 \pm 33.460$ & $2.843 \pm 1.028$ & $4.429 \pm 1.405$ & $23.12 \pm 12.70$ \\
Rajasthan & 1527 & $168.13 \pm 31.40$ & $113 \pm 47.44$ & $45.10 \pm 10.75$ & $100.50 \pm 27.71$ & - & - & - \\
Andhra Pradesh & 1922 & $175.3 \pm 34.6$ & $132 \pm 60.9$ & $47.2 \pm 10.4$ & $101.1 \pm 3.0$ & $2.4 \pm 1$ & $4.15 \pm 1.13$ \\
Bengal & 1396 & $189.7 \pm 33.3$ & $132 \pm 42.2$ & $52.9 \pm 10.1$ & $115.6 \pm 29.8$ & - & - & $21.1 \pm 6.5$ \\
Maharashtra & 751 & $198 \pm 37.16$ & $119 \pm 53.27$ & $47 \pm 11.13$ & $121 \pm 29.39$ & - & $4.4 \pm 1.13$ & - \\
Venezuela & 434 & $191.96 \pm 46$ & $130.50 \pm 102.53$ & $44.27 \pm 12.12$ & $122.10 \pm 38.40$ & - & - & $26.10 \pm 20.50$ \\
Nepal & 454 & $184.0 \pm 50.7$ & $147.4 \pm 88.7$ & $45.0 \pm 11.7$ & $111.9 \pm 42.0$ & - & - & - \\
\hline
\end{tabular}

TC: Total Cholesterol; TG: Triglycerides; HDL-C: High Density Lipoprotein Cholesterol; LDL-C: Low Density Lipoprotein Cholesterol; VLDL: Very Low Density Lipoprotein. Data are expressed as mean \pm SD.

in the lipid profile [11, 25], we had obtained significant difference between the risk profiles of males and females of the study population. The females were having superior CVD risk factor profile due to the estrogenic protective effect, which was found to be lost in the 5th to the 6th decade of life.

Natural ageing induced elevation in CVD risk factors was observed in the study population; however, surprisingly, the mean value of HDL was found to increase steadily after the 3rd decade with the increasing age in both genders which is in accordance with other studies (Andhra Pradesh). A significant finding reflected from decade-wise analysis is the loss of premenopausal protection in females in the 5 th to 6 th decade of life as the mean values of serum Total Cholesterol, LDL-C, SBP, and BMI were higher in females of 50-59 age group as compared to their male counterpart of the same age group [11, 12]. Several epidemiological studies have shown the interplay between multiple risk factors of CVD such as dyslipidemia, diabetes, BMI, and hypertension [26, 27]. In concordance with our study, the coexistence of these risk factors in Gujarati population has been reported by others also [28]. One of the most striking outcomes of the study is the relatively low level of HDL-C in Gujaratis compared to Rajasthani, Andhra Pradesh, Bengali, Maharashtrian, Venezuelan, and Nepalese population $[10,12,29-32]$. All of these studies 
were undertaken on apparently healthy population similar to ours. Prajapati et al. had shown great degree of association between higher carotid intima thickness and low HDL-C [33] levels in Gujarati children, making HDL-C one of the strongest predictors of $\mathrm{CAD}$ in the studied community. We have observed the alarmingly high proportion of population being unaware of the CVD risk factors prevalence.

Representative percentile reference data of modifiable risk factors of CVD are highly required for the framing of appropriate diagnostic and preventive policies. The values provided in the current study could be effectively used as reference values that are specific for Gujarati population. The strengths of the study are the well-defined percentile values of modifiable risk factors, larger population size, standard laboratory techniques, and participants from different regions of Gujarat state. However, the study also possesses some limitations such as lack of information regarding the history of genetics, diet, and stress, daily consumption of alcohol and tobacco of the study population.

\section{Conclusion}

The outcome of the current study will contribute significantly to standardization of the reference values of various lipids, glucose, blood pressure, and BMI which are of clinical importance and will help in the designing of better diagnostic strategies.

\section{Conflict of Interests}

The authors declare that there is no conflict of interests regarding the publication of this paper.

\section{References}

[1] K. S. Reddy and S. Yusuf, "Emerging epidemic of cardiovascular disease in developing countries," Circulation, vol. 97, no. 6, pp. 596-601, 1998.

[2] K. E. North, B. V. Howard, T. K. Welty et al., "Genetic and environmental contributions to cardiovascular disease risk in American Indians the strong heart family study," American Journal of Epidemiology, vol. 157, no. 4, pp. 303-314, 2003.

[3] R. Gupta, S. Guptha, K. K. Sharma, A. Gupta, and P. Deedwania, "Regional variations in cardiovascular risk factors in India: India heart watch," World Journal of Cardiology, vol. 4, no. 4, pp. 112-120, 2012.

[4] H. Zeljko, T. Škarić-Jurić, N. S. Narančić et al., "Traditional CVD risk factors and socio-economic deprivation in Roma minority population of Croatia," Collegium Antropologicum, vol. 32, no. 3, pp. 667-676, 2008.

[5] R. Carmena, P. Duriez, and J.-C. Fruchart, "Atherogenic lipoprotein particles in atherosclerosis," Circulation, vol. 109, no. 23, supplement 3, pp. III-2-III-7, 2004.

[6] I. Shai, E. B. Rimm, S. E. Hankinson et al., "Multivariate assessment of lipid parameters as predictors of coronary heart disease among postmenopausal women: potential implications for clinical guidelines," Circulation, vol. 110, no. 18, pp. 28242830, 2004.
[7] R. Gupta, S. Guptha, A. Agrawal, V. Kaul, K. Gaur, and V. P. Gupta, "Secular trends in cholesterol lipoproteins and triglycerides and prevalence of dyslipidemias in an urban Indian population," Lipids in Health and Disease, vol. 7, no. 1, article 40, 2008.

[8] A. S. Foulkes, D. A. Wohl, I. Frank et al., "Associations among race/ethnicity, ApoC-III genotypes, and lipids in HIV-1infected individuals on antiretroviral therapy," PLoS Medicine, vol. 3, no. 3, pp. 337-347, 2006.

[9] National Heart, Lung, and Blood Institute, “Third report of the National Cholesterol Education Program (NCEP) expert panel on detection, evaluation, and treatment of high blood cholesterol in adults (Adult Treatment Panel III) final report," Circulation, vol. 106, no. 25, pp. 3143-3421, 2002.

[10] D. Yadav, M. Gupta, S. Mishra, and P. Sharma, "Reference interval for lipid profile in North Indian population from Rajasthan according to various partitioning criteria," Clinica Chimica Acta, vol. 426, pp. 145-151, 2013.

[11] M. Das and M. Saikia, "Stimation of reference interval of lipid profile in Assamese population," Indian Journal of Clinical Biochemistry, vol. 24, no. 2, pp. 190-193, 2009.

[12] T. Malati and M. R. U. Mahesh, "Reference intervals for serum total cholesterol, HDL-cholesterol, LDL-cholesterol, triglycerides, Lp (a), apolipoprotein A-I, A-II, B, C-II, C-III, and E in healthy South Indians from Andhra Pradesh," Indian Journal of Clinical Biochemistry, vol. 24, no. 4, pp. 343-355, 2009.

[13] M. P. M. de Maat, F. Green, P. de Knijff, J. Jespersen, and C. Kluft, "Factor VII polymorphisms in populations with different risks of cardiovascular disease," Arteriosclerosis, Thrombosis, and Vascular Biology, vol. 17, no. 10, pp. 1918-1923, 1997.

[14] J. K. Cruickshank, J. Cooper, M. Burnett, J. Macduff, and U. Drubra, "Ethnic differences in fasting plasma C-peptide and insulin in relation to glucose tolerance and blood pressure," The Lancet, vol. 338, no. 8771, pp. 842-847, 1991.

[15] S. R. Joshi, B. Saboo, M. Vadivale et al., "Prevalence of diagnosed and undiagnosed diabetes and hypertension in India-results from the Screening India's Twin Epidemic (SITE) study," Diabetes Technology and Therapeutics, vol. 14, no. 1, pp. 8-15, 2012.

[16] D. V. Bala, I. L. A. N. Bodiwala, D. D. Patel, and P. M. Shah, "Epidemiological determinants of tobacco use in Gujrat State, India," Indian Journal of Community Medicine, vol. 31, no. 3, pp. 173-176, 2006.

[17] P. M. McKeigue, B. Shah, and M. G. Marmot, "Relation of central obesity and insulin resistance with high diabetes prevalence and cardiovascular risk in South Asians," The Lancet, vol. 337, no. 8738, pp. 382-386, 1991.

[18] C. I. Jhala, U. V. Shah, T. K. Shah, B. K. Naik, and J. D. Dafda, "A study of serum lipid profile part-1: establishment of normal reference values of serum lipid levels in healthy vegetarian population of Gujarat," Indian Journal of Clinical Biochemistry, vol. 13, no. 1, pp. 1-7, 1998.

[19] Indian Consensus Group, "Indian consensus for prevention of coronary artery disease: a joint scientific statement of Indian society of hypertension and International College of Nutrition," Journal of Nutritional and Environmental Medicine, no. 6, pp. 309-318, 1996.

[20] World Health Organization, The World Health Report: 2002: Reducing Risks, Promoting Healthy Life: Overview, World Health Organization, Geneva, Switzerland, 2002.

[21] S. Kanjilal, J. Shanker, V. S. Rao et al., "Prevalence and component analysis of metabolic syndrome: an Indian atherosclerosis 
research study perspective," Vascular Health and Risk Management, vol. 4, no. 1, pp. 189-197, 2008.

[22] H. Pandya, J. D. Lakhani, and N. Patel, "Obesity is becoming synonym for diabetes in rural areas of india also an alarming situation," International Journal of Biological and Medical Research, vol. 2, no. 2, pp. 556-560, 2011.

[23] A. Joshi, P. Bhugra, J. Lakhani, and S. Desai, "Body mass index and central obesity in hypertensive patients," Gujarat Medical Journal, vol. 61, no. 3, pp. 33-36, 2004.

[24] C. Jhala, P. Joshi, T. Shah, and B. Naik, "Establishment of normal lipid reference values in healthy vegetarian population of rural western \& northern Gujarat and comparison with available similar data of other parts of India," Gujarat Medical Journal, vol. 64, no. 2, pp. 25-31, 2009.

[25] V. Kaur, M. Verma, A. Kaur, S. Gupta, and K. Singh, "To establish the reference intervals of lipid profile in Punjab," Indian Journal of Clinical Biochemistry, vol. 27, no. 3, pp. 290295, 2012.

[26] J. F. Price, P. I. Mowbray, A. J. Lee, A. Rumley, G. D. O. Lowe, and F. G. R. Fowkes, "Relationship between smoking and cardiovascular risk factors in the development of peripheral arterial disease and coronary artery disease; Edinburgh Artery study," European Heart Journal, vol. 20, no. 5, pp. 344-353, 1999.

[27] D. Betteridge, "The interplay of cardiovascular risk factors in the metabolic syndrome and type 2 diabetes," European Heart Journal Supplements, vol. 6, supplement G, pp. G3-G7, 2004.

[28] K. H. Sharma, S. Sahoo, K. H. Shah et al., "Are Gujarati Asian Indians 'older' for their 'vascular age' as compared to their 'chronological age'?” Quarterly Journal of Medicine, vol. 105, no. 2, pp. 105-112, 2014.

[29] T. F. Ashavaid, A. A. Kondkar, S. P. Todur, A. J. Dherai, J. Morey, and R. Raghavan, "Lipid, lipoprotein, apolipoprotein and lipoprotein (a) levels: reference intervals in a healthy Indian population," Journal of Atherosclerosis and Thrombosis, vol. 12, no. 5, pp. 251-259, 2005.

[30] K. Goswami and A. Bandyopadhyay, "Lipid profile in middle class Bengali population of Kolkata," Indian Journal of Clinical Biochemistry, vol. 18, no. 2, pp. 127-130, 2003.

[31] V. J. Bermúdez, L. M. Bello, A. Naguib et al., "Lipid profile reference intervals in individuals from Maracaibo, Venezuela: an insight from the Maracaibo City Metabolic Syndrome prevalence study," Revista Latinoamericana de Hipertension, vol. 7, no. 2, pp. 24-34, 2011.

[32] Y. R. Limbu, S. K. Rai, K. Ono et al., "Lipid profile of adult Nepalese population," Nepal Medical College Journal, vol. 10, no. 1, pp. 4-7, 2008.

[33] J. Prajapati, S. Dani, S. Jain et al., "AS-209: risk factor profile for coronary artery disease in very young gujarati population," The American Journal of Cardiology, vol. 103, no. 9, supplement, p. 90B, 2009. 


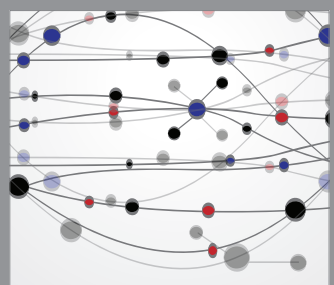

The Scientific World Journal
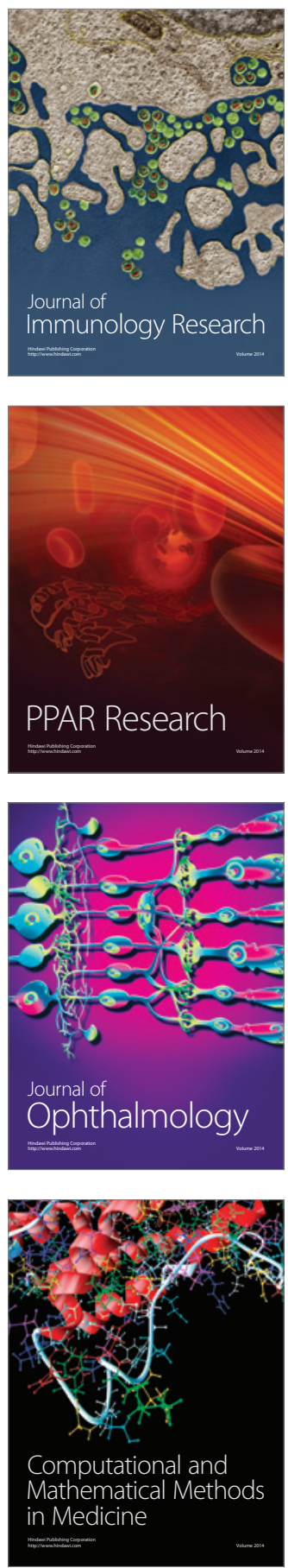

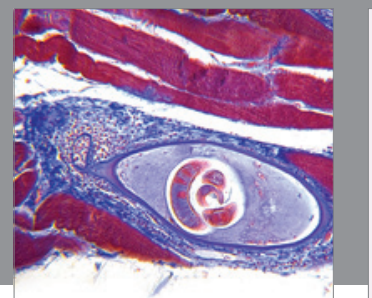

Gastroenterology

Research and Practice
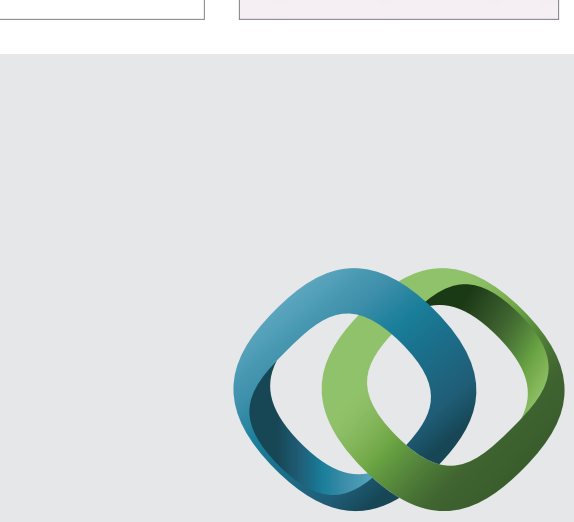

\section{Hindawi}

Submit your manuscripts at

http://www.hindawi.com
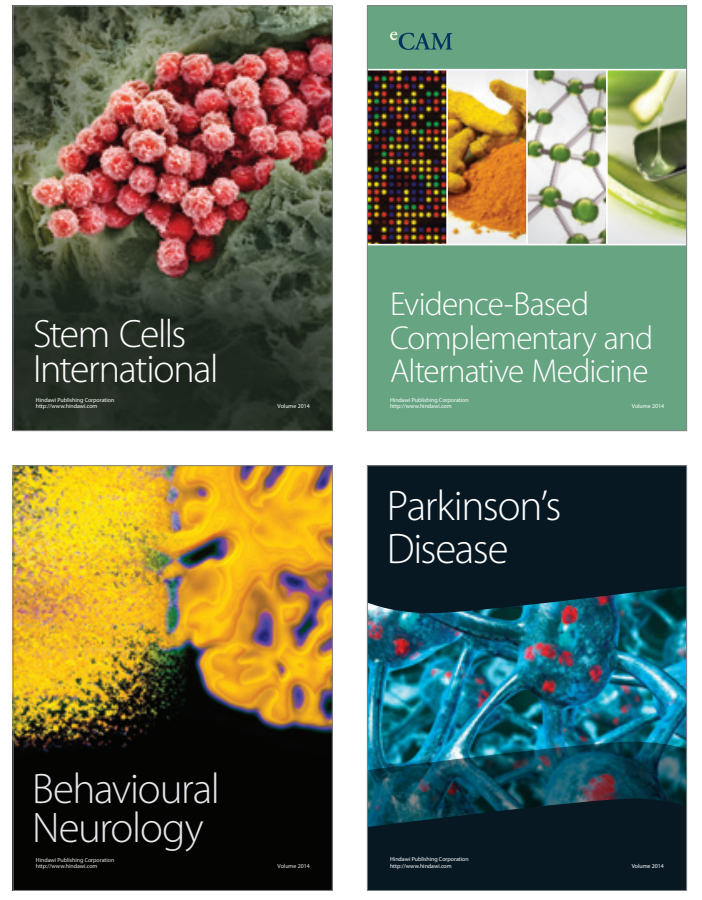
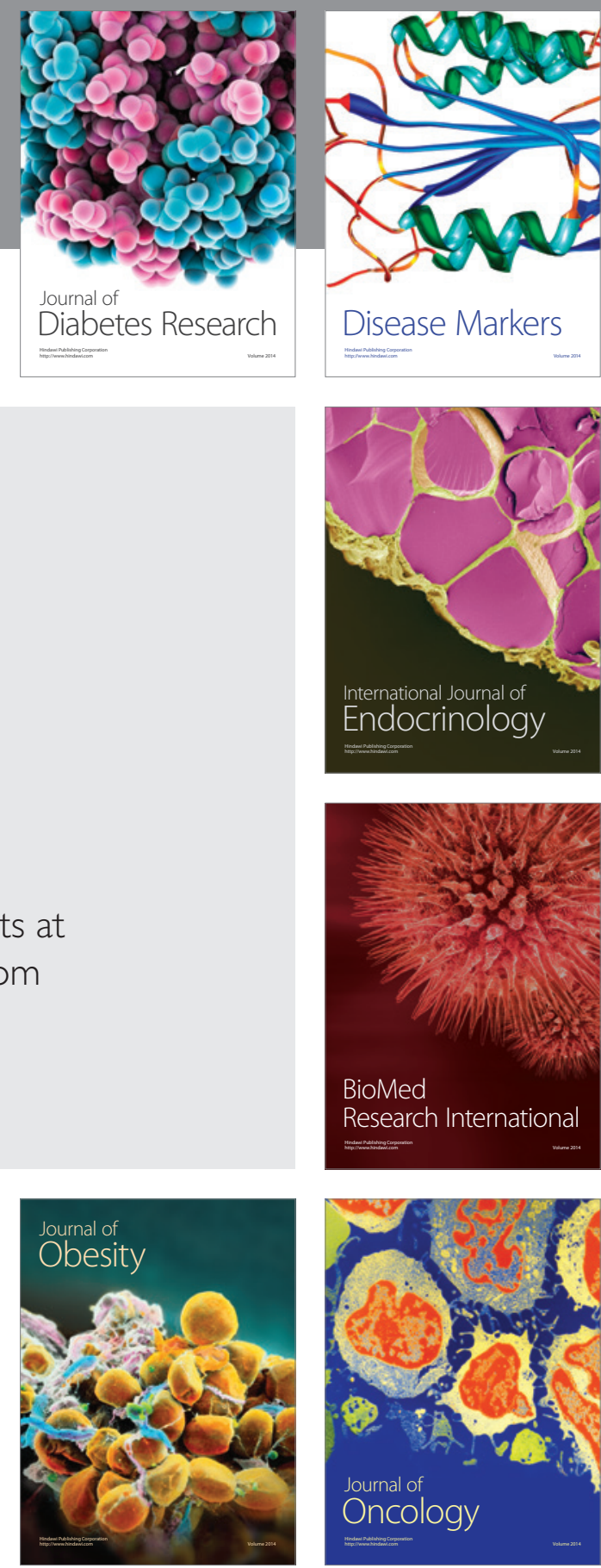

Disease Markers
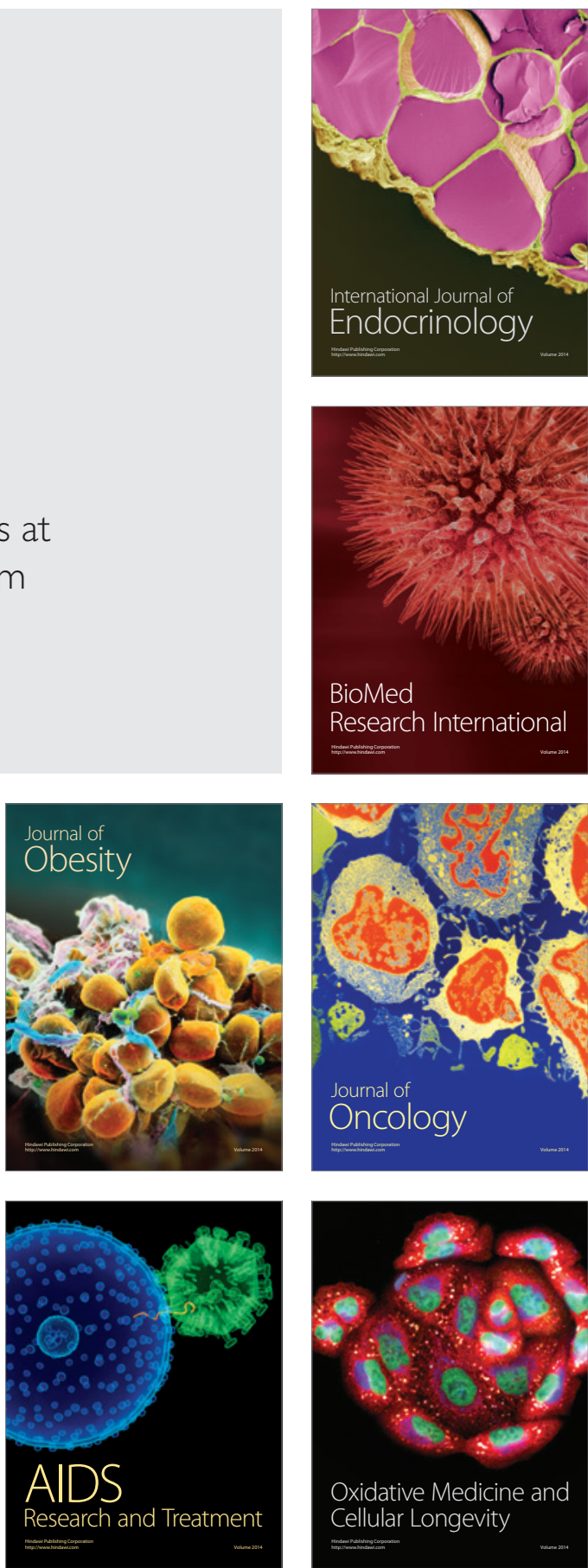